\title{
Mesangial IgA deposits indicate pathogenesis of anti-glomerular basement membrane disease
}

\author{
AIFENG WANG $^{1}$, YONGPING WANG ${ }^{2}$, GUOBAO WANG $^{2}$, ZHANMEI ZHOU $^{2}$, \\ ZHANG XUN $^{2}$ and XIAOHUI TAN ${ }^{1}$ \\ ${ }^{1}$ Institute of Forensic Medicine, Preclinical Medical College, and ${ }^{2}$ Division of Nephrology, Nanfang Hospital, \\ Southern Medical University, Guangzhou, Guangdong, P.R. China
}

Received November 12, 2011; Accepted February 10, 2012

DOI: $10.3892 / \mathrm{mmr} .2012 .809$

\begin{abstract}
Anti-glomerular basement membrane (anti-GBM) disease is characterized by crescentic glomerulonephritis with immunoglobulin $\mathrm{G}$ ( $\mathrm{IgG}$ ) autoantibodies to the noncollagenous (NC1) domain of $\alpha 3$ (IV) collagen presenting along the GBM. The patient clinically manifests with rapidly progressive glomerulonephritis (RPGN) with pulmonary hemorrhage (Goodpasture syndrome). In rare cases, other immunocomplexes of $\operatorname{Ig} \mathrm{A}$ or IgM are involved, but their specificities have not been determined. We report a rare case of a 31-year-old female who was diagnosed as having anti-GBM disease with extensive $\operatorname{IgA}$ deposits in the mesangium. This patient presented heavy hematuria, proteinuria with increasing creatinine, but no lung hemorrhage. Renal biopsy showed crescentic glomerulonephritis (type I) with strong IgA (3+) as lump and branch shape. Therapies with pulse methylprednisolone, plasmapheresis and cyclophosphamide administration were less effective. This case is different from the present type I crescentic glomerulonephritis and the specificity of IgA deposits may implicate the pathogenesis of anti-GBM disease.
\end{abstract}

\section{Introduction}

Anti-glomerular basement membrane (anti-GBM) disease is characterized by circulating anti-GBM antibodies and mesangial deposits of these antibodies. It is associated with extensive crescent formation and rapidly progressive glomerulonephritis (RPGN). When it is associated with pulmonary hemorrhage, it is called Goodpasture syndrome (1). Smooth linear deposits of anti-GBM antibodies identified by immunofluorescence from renal biopsies are hallmarks of the disease, and serum circulating anti-GBM antibodies can be detected

Correspondence to: Dr Yongping Wang, Division of Nephrology, Nanfang Hospital, Southern Medical University, No. 1838 Northern Guangzhou Street, Guangzhou, Guangdong 510515, P.R. China E-mail: wang-yongping@hotmail.com

Key words: anti-glomerular basement membrane disease, crescentic glomerulonephritis, IgA, pathogenesis using enzyme-linked immunosorbent assay (ELISA). Although relatively rare, anti-GBM disease is a paradigm for antibodymediated glomerular injury, since the autoantibodies and their target autoantigen have been extensively characterized (2). Most patients have immunoglobulin $\mathrm{G}$ ( $\mathrm{IgG}$ ) autoantibodies against the non-collagenous (NC1) domain of the $\alpha 3$ (IV) collagen chain (the Goodpasture autoantigen), which is present in the GBM and the alveolar basement membrane $(3,4)$. Here, we describe a rare case of anti-GBM disease with extensive IgA deposits in the mesangium, which is similar to indications of $\operatorname{IgA}$ nephropathy. The specificity of the $\operatorname{IgA}$ antigen deposits may be implicated in the pathogenesis or prognosis of anti-GBM disease.

\section{Case report}

A 31-year-old female was admitted to the nephrology ward of Nanfang Hospital with swelling of the face and lower limbs, together with heavy hematuria (4+) and proteinuria ( $3.76 \mathrm{~g} / 24 \mathrm{~h})$ one week prior. Physical examination revealed that the patient was in no acute distress and that she was afebrile with an elevated blood pressure of $113 / 75 \mathrm{mmHg}$. Her cardiac, pulmonary and abdominal examinations were normal. Slight umbilication edema of the lower extremities was present. No rashes or lymph nodes were noted. Laboratory investigations showed normal blood biochemical levels but a decreased kidney function with a creatinine level of $287 \mu \mathrm{mol} / 1$, blood urea nitrogen of $9.7 \mathrm{mmol} / \mathrm{l}$ and uric acid of $437 \mu \mathrm{mol} / 1$. Serum quantitations of self-antibodies showed normal levels of total antineutrophil cytoplasmic autoantibody (ANCA, 47.8 AAU/ ml), P-ANCA/MPO (22.6 AAU/ml), C-ANCA (27.0 AAU/ $\mathrm{ml}$ ), antinuclear antibodies (ANA, $23.9 \mathrm{U} / \mathrm{ml}$ ), ds-DNA (33 U/ $\mathrm{ml}), \mathrm{Sm}(6.0 \mathrm{U} / \mathrm{ml})$ and RNP $(12.6 \mathrm{U} / \mathrm{ml})$, but high amounts of anti-GBM antibody (93.5 RU/ml). There was no hepatitis B virus $\mathrm{S}$ antigen or anti-hepatitis $\mathrm{C}$ virus antibodies detected. Ultrasound showed slight enlargment of both kidneys with increased parenchymal echogenicity, and seroperitoneum was also found. The patient was evaluated using CT scan for pleural effusion and pachynsis pleurae bilaterally.

Renal biopsy yielded cortex containing 17 glomeruli without global or segmental sclerosis. Moderate to severe proliferation of the mesangial areas were found asystematically with small amounts of immune complex deposit (Fig. 1A and B). A total 


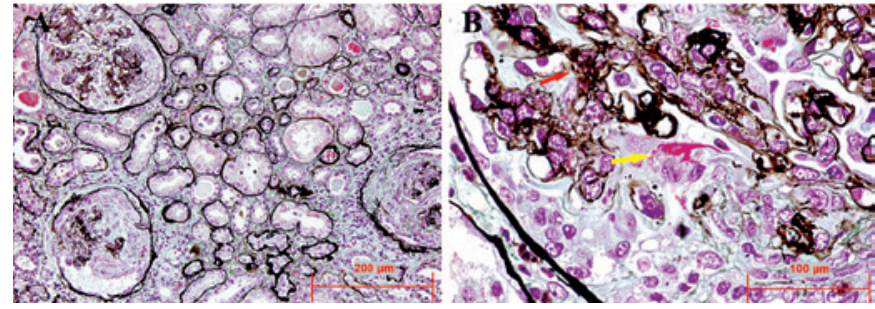

Figure 1. Active necrotizing crescentic glomerulonephritis was present in the renal biopsy by Masson's trichrome covering with silver staining. (A) Large crescents, (B, yellow arrow) necrosis and (red arrow) philo-fuchsin complex were also noted. Scale bar, 200 and $100 \mu \mathrm{m}$ (A and B, respectively).
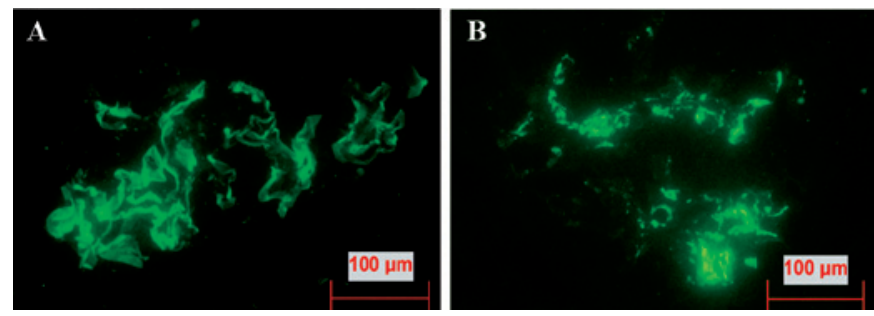

Figure 2. Direct immunofluorescence was shown in the renal biopsy specimen. (A) Linear fixation of IgG was shown along the GBM, while the deposition of IgA was exhibited, (B) predominantly within the mesangial regions of glomeruli. Scale bar, $100 \mu \mathrm{m}$.

of 14 crescents were found, including 5 large-cellular, 6 largecellular/fibrous, 2 small-cellular and 1 small-cellular/fibrous. One glomerulus had an adhesion of the glomerular tuft to Bowman's capsule and obvious capillary necrosis. Moderate interstitial inflammation and fibrosis, and tubular atrophy with proteinic and red cell casts were present. The small arteries showed no evidence of necrosis or thickening of their walls. By Masson or PAS staining, no spikes, mesangial interposition or thickening of the GBM could be demonstrated. Direct immunofluorescence showed strong (3+) linear capillary loop staining for human $\mathrm{IgG}$, together with mesangial staining for IgA (3+), IgM (1+) and C3 (2+) (Fig. 2A and B). The GBM was relatively normal, but many electron dense deposits were found in the mesangial space (Fig. 3). Extensive podocyte effacements were noted.

The patient received three courses of intravenous pulse therapy with methylprednisolone $(0.5 \mathrm{~g} /$ day $)$, followed by oral cyclophosphamide ( $1 \mathrm{mg} / \mathrm{kg} /$ day $)$ and prednisone $(1 \mathrm{mg} / \mathrm{kg} /$ day $)$. Plasmapheresis was performed 10 times due to the morphology of the biopsy specimen and the gradual progression of the disease. After 29 days of therapy, the amounts of anti-GBM antibody decreased to a normal level (19.7 RU/ml), while her serum creatinine remained at $375 \mu \mathrm{mol} / 1$. During treatment, the patient showed no signs of pulmonary hemorrhage or other serious symptoms.

\section{Discussion}

We report a rare case of $\operatorname{Ig} \mathrm{A}$ deposits in the mesangium of a patient with anti-GBM disease. The sequential or simultaneous presentation of anti-GBM disease with IgA nephropathy has been infrequently described. This raises the question of

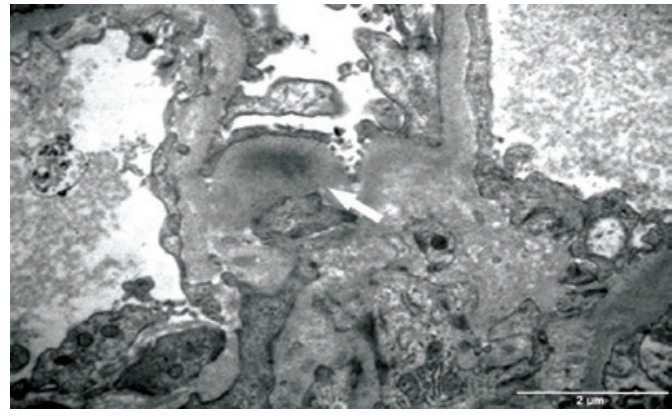

Figure 3. Electron-dense deposits were noted within the mesangial regions by electron microscopy (arrow). Scale bar, $2 \mu \mathrm{m}$.

a possible connection between the two diseases. Primary IgA nephropathy is an immune complex-mediated glomerulonephritis defined immunohistologically by the presence of glomerular IgA deposits accompanied by a variety of histopathologic lesions (5). It is suggested that familial IgA nephropathy is a complex disease initiated by one or more genes in combination with environmental conditions (6). Anti-GBM disease is characterized by the occurrence of glomerulonephritis and pulmonary hemorrhage caused by antibodies reactive to the glomerular and alveolar basement membrane. Ongoing glomerular pathological changes may alter GBM components, triggering an autoimmune reaction to modified antigens, as proposed for the association between anti-GBM disease and ANCA glomerulonephritis. We reviewed the history of this patient, but did not discover related symptoms of IgA nephropathy, such as chronic cough, expectoration, upper respiratory tract infection or coeliodynia.

The patient presented with no pulmonary hemorrhage, or evidence of ANCA-associated glomerulonephritis. A lung biopsy was not performed nor was sputum obtained, so involvement of the lung could not be documented. The patient was evaluated by CT scan, and pathologic changes in the lung may have been ignored. We aim to ascertain whether there may be a relationship between $\operatorname{Ig}$ A deposits and anti-GBM disease, or whether they are co-effectors. Trpkov et al (7) previously reported that a patient wth anti-GBM disease presented with Ig A nephropathy during his posttransplant course, yet the association between these two diseases was unclear. Another report study revealed that anti-GBM disease was associated with inflammatory bowel disease (8). The author concluded that anti-GBM nephritis was a potential extraintestinal manifestation of inflammatory bowel disease that occurred in the setting of active bowel inflammation. Anti-GBM disease is also demonstrated to simultaneously exist with membranous glomerulonephritis in a rare entity (9), although it is difficult to describe preceding or following recovery from these two diseases. Study of our case may provide insight into pathophysiologic mechanisms, including the possibility of increased antigen synthesis, exposure of cryptic epitopes, or capping and shedding of antigen-antibody complexes.

In conclusion, we report a case of anti-GBM disease with $\operatorname{Ig} \mathrm{A}$ deposits in the mesangium, which is distinct from the former types. The patient was refractory to conventional therapy with cyclophosphamide and steroids and needed further investigation to identify the characteristics. 


\section{Acknowledgements}

The authors would like to thank Professor John M. Basgen for his insightful suggestions for this report.

\section{References}

1. Borza DB, Chedid MF, Colon S, et al: Recurrent Goodpasture's disease secondary to a monoclonal $\operatorname{Ig} \mathrm{A} 1-\kappa$ antibody autoreactive with the $\alpha 1 / \alpha 2$ chains of type IV collagen. Am J Kidney Dis 45: 397-406, 2005.

2. Borza DB, Neilson EG and Hudson BG: Pathogenesis of Goodpasture syndrome: a molecular perspective. Semin Nephrol 23: 522-531, 2003.

3. Borza DB, Netzer KO, Leinonen A, et al: The Goodpasture autoantigen. Identification of multiple cryptic epitopes on the $\mathrm{NC1}$ domain of the alpha3(IV) collagen chain. J Biol Chem 275: 6030-6037, 2000.
4. Hellmark T, Segelmark M, Unger C, et al: Identification of a clinically relevant immunodominant region of collagen IV in Goodpasture disease. Kidney Int 55: 936-944, 1999.

5. Emancipator SN: IgA nephropathy: morphologic expression and pathogenesis. Am J Kidney Dis 23: 451-462, 1994.

6. Donadio JV and Grande JP: IgA nephropathy. N Engl J Med 347: 738-748, 2002.

7. Trpkov K, Abdulkareem F, Jim K and Solez K: Recurrence of anti-GBM antibody disease twelve years after transplantation associated with de novo IgA nephropathy. Clin Nephrol 49: 124-128, 1998

8. Shaer AJ, Stewart LR, Cheek DE, et al: IgA antiglomerular basement membrane nephritis associated with Crohn's disease: a case report and review of glomerulonephritis in inflammatory bowel disease. Am J Kidney Dis 41: 1097-1109, 2003.

9. Troxell ML, Saxena AB and Kambham N: Concurrent antiglomerular basement membrane disease and membranous glomerulonephritis: a case report and literature review. Clin Nephrol 66: 120-127, 2006. 